\title{
Chairman's summary
}

There is no point in attempting to give a detailed summary of two whole days of papers and discussions. Instead I will offer a brief personal impression of some points that I think we have learned.

To use enzyme assays intelligently in medicine it is necessary to keep up with advances in basic biochemistry.

The problems of standardization are great, especially in relation to automated methods. Conversion factors are often a delusion.

Many methods are not ideal and perhaps they never can be; this is partly due to the varying proportion in serum of isoenzymes with different analytical properties.

Much of the disagreement between results obtained in different centres is due to methodological differences, often undefined.

It is yet to be decided whether the advantages of precision that may be realized by automation at central laboratories outweigh the disadvantages of delay in presenting specimens for analysis.

More work must be done on the subcellular distribution of tissue enzymes and their changes in disease; it is not yet known whether histochemistry is the final answer.

There is probably no serum enzyme or isoenzyme analysis available which is absolutely organ-specific.

Looking for a single enzyme deficiency is usually more profitable than looking for a single enzyme excess.

Though analysis of several enzymes usually gives more information on a patient than does a single analysis, repeated doubling of the number of enzymes tested does not continue to double the diagnostic specificity.

D. N. BARON 